\title{
MEDIO AMBIENTE, \\ DESARROLLO SOSTENIBLE \\ Y ESCALAS DE SUSTENTABILIDAD
}

\author{
Ángel Paniagua \\ Instituto de Economía y Geografía. CSIC. Madrid \\ Eduardo Moyano ${ }^{1}$ \\ IESA-Andalucía. CSIC. Córdoba
}

\section{RESUMEN}

El presente artículo tiene dos partes. En la primera se analiza la complejidad y las múltiples dimensiones de concepto de sustentabilidad. La perspectiva que se adopta se fundamenta en que no existe una opinión clara y universalmente admitida sobre el concepto y, en consecuencia, una de sus principales características es su vaguedad. En la segunda parte se estudian las diferentes opciones de desarrollo rural sustentable desde una perspectiva global y se proponen diferentes escalas de sustentabilidad rural.

\section{INTRODUCCIÓN}

La aparición y difusión del término "desarrollo sostenible o sustentable»² puede decirse que ha ido paralela a un mayor interés por el medio ambiente en

1 Agradecemos a Fernando Garrido sus comentarios y, en especial, las definiciones que nos ha facilitado sobre sustentabilidad y desarrollo sostenible, que se incluyen como anejo a este artículo, y a Cristóbal Gómez Benito sus anotaciones a una versión inicial. Los autores también quieren manifestar su reconocimiento a un evaluador anónimo por sus sugerencias de detalle que han contribuido a enriquecer el presente texto. Por último, indicar que este artículo forma parte del proyecto de la DGES PB95-0076.

${ }_{2}^{2}$ Los términos sostenible y sustentable se suelen utilizar indistintamente como traducción al español del término inglés sustainable. Mientras que en Latinoamérica se utiliza más frecuentemente la expresión «desarrollo sustentable», en España es más común la de «desarrollo sosteni-

\section{Reis}


la sociedad. Un mayor interés o preocupación que no se dejó sentir en las décadas de los cuarenta y cincuenta, cuando los objetivos de desarrollo se centraban en el crecimiento económico, sin reparar en el elevado coste energético de los nuevos procesos ni en el deterioro o reducción de ciertos recursos naturales. En los años sesenta, sin embargo, la constatación de los primeros problemas medioambientales de importancia sirvió para que empezaran a denunciarse las amenazas que sobre el entorno natural tenía o podía tener el acelerado ritmo de expansión que se estaba llevando a cabo en los países industrializados.

No es, sin embargo, hasta finales de los años sesenta y principios de los setenta que el tema del medio ambiente comienza a expandirse de un modo general, alcanzando a esferas más amplias de la sociedad. En este sentido, se hacen más conocidas algunas de las organizaciones ecologistas nacidas en los años sesenta, como la WWF (World Wild Fund) en 1961, o ADENA (Asociación para la Defensa de la Naturaleza) en 1968, mientras que hacen su aparición en los setenta otras asociaciones de importancia internacional como Greenpeace (1971), en Canadá, o Amigos de la Tierra (1979). Si bien estas organizaciones no tenían todavía gran influencia en la opinión pública ni sus puntos de vista se tenían demasiado en cuanta en los procesos de toma de decisiones, su aparición era ya reflejo de una mayor inquietud social por las cuestiones ambientales.

En el terreno académico, la publicación de los dos informes elaborados por el MIT (Massachusetts Institute of Technology) al Club de Roma en 1972 (Limits to growth) y 1974 (Mankind at the Turning Point) también ponía de manifiesto, por medio de unos modelos de simulación, los efectos negativos sobre el medio ambiente causados por el modelo de desarrollo productivista vigente en los países industrializados. Las primeras referencias a la necesidad de un desarrollo sostenible ya tenían cabida en los debates académicos sobre los modelos de desarrollo, debates que, si bien no tuvieron inicialmente unos resultados esperanzadores, contribuyeron a despertar la preocupación por los problemas ecológicos entre la población de los países industrializados.

En el plano institucional y a nivel internacional, también los años setenta son testigo de los primeros encuentros y reuniones para discutir sobre el medio ambiente a escala global. La declaración de 1970 como Año de la Protección de la Naturaleza a instancias del Consejo de Europa, o la celebración de la Primera Conferencia Mundial sobre Medio Ambiente, celebrada en Estocolmo en 1972 y auspiciada por la ONU, son pruebas palpables de que el medio ambiente comenzaba a tener eco en las instituciones supranacionales. En la Unión Europea (por aquel entonces, Comunidad Económica Europea) también se pueden citar por aquella época acciones administrativas de esa índole, como la aprobación del Primer Programa de Acción sobre Medio Ambiente (1973-1976). En la segunda mitad de la década de los setenta, los problemas

ble». En este trabajo utilizaremos el término sostenible como adjetivo para calificar un determinado modelo de desarrollo (por ej.: desarrollo sostenible), pero usaremos el término sustentabilidad cuando lo utilicemos como sustantivo (por ej.: el enfoque de la sustentabilidad). 
medioambientales pasaron, sin embargo, a un segundo plano. La recesión económica, el crecimiento del desempleo y el déficit financiero del sector público constituían los principales problemas para los gobiernos occidentales.

Durante los años ochenta el interés por lo ecológico iba ganando, de nuevo, relevancia entre la población. Esta mayor preocupación social por el medio ambiente se vio estimulada por la propia constatación de la gravedad de algunos de los problemas medioambientales (crecimiento demográfico incontrolado, aglomeraciones urbanas, contaminación atmosférica, agujero de la capa de ozono, impacto de algunos desastres ecológicos como la explosión de la central nuclear de Chernobil en Ucrania o el hundimiento del Exxon Valdez en las costas de Alaska, etc.). El avance del conocimiento científico, una mejor comprensión de las causas de algunos de los fenómenos desencadenantes de procesos contaminantes y, sobre todo, el mayor protagonismo de los medios de comunicación en la difusión de noticias relacionadas con este tema, son factores que deben tenerse en cuenta a la hora de explicar el creciente interés por el medio ambiente en esa década.

Nuevos grupos ecologistas nacían en ese período y los ya existentes aumentaban su número de socios y adquirían un protagonismo mayor del que habían disfrutado hasta entonces. Las reuniones internacionales para debatir sobre el medio ambiente se multiplican (Conferencia Mundial sobre Medio Ambiente, 1982; Cumbre de Río, 1992...), estableciéndose, además, acuerdos entre los gobiernos de algunas naciones sobre la necesidad de tomar medidas para la conservación de los recursos naturales. En el ámbito de la Unión Europea (UE) se continuó con los programas de acción sobre medio ambiente y con las regulaciones para controlar la contaminación, principalmente la de origen industrial. En lo que concierne al sector agrario, la Comisión de la UE publicó en 1985 un documento titulado Perspectivas de la PAC (más conocido como Libro Verde), en el que se destacaba "la necesidad de que la política agraria tuviera más en cuenta la política medioambiental, tanto en lo que respecta a la prevención de las actividades nocivas, como en relación a la promoción de prácticas beneficiosas para el medio ambiente».

También en las esferas científicas el medio ambiente aparece de forma sistemática como objeto de debate. Economistas, biólogos, sociólogos, planificadores, etc., eligen con frecuencia el medio ambiente, de forma directa o indirecta, como tema central en sus estudios y proyectos de investigación. Producto de esta inquietud es la aparición de nuevos libros e informes, como el libro de Donella y Dennis Meadows Más allá de los límites del crecimiento — publicado veinte años después de su primer informe al Club de Roma-, en el que se confirman algunas de las predicciones que ya se hicieron sobre las consecuencias del modelo de desarrollo productivista, y el conocido Informe Brundtland (1987) (Nuestro futuro común), publicado por la Comisión Mundial del Medio Ambiente y el Desarrollo, libros todos ellos destacables por el impacto que han tenido no sólo en los círculos científicos, sino también en la opinión pública y los medios de comunicación. Resultado de los debates en torno a estos temas, 
donde se han vertido críticas al modelo de desarrollo productivista y se han propuesto nuevas formas de desarrollo que tengan más en cuenta la conservación de los recursos naturales y la preservación del medio ambiente, ha sido la generalización de términos como sustentabilidad y desarrollo sustentable, términos que han sido adoptados prácticamente a nivel mundial como principios directores de las políticas públicas.

\section{EL CONCEPTO DE DESARROLLO SOSTENIBLE}

Desde su primera aparición pública de importancia en la Estrategia Mundial de Conservación de 1980, el término desarrollo sostenible ha venido a representar la más conocida y divulgada aproximación al problema de la interacción entre las actividades económicas y el medio ambiente. Un factor de especial relevancia para su difusión y aceptación por la comunidad internacional fue la publicación, en 1987, del ya citado Informe Brundtland de la Comisión Mundial del Medio Ambiente y del Desarrollo (CMMAD), que proclamó el desarrollo sostenible como el objetivo central de la política económica. Según esta Comisión, el desarrollo sostenible sería «aquel que es capaz de cubrir las necesidades del presente sin comprometer las posibilidades de las futuras generaciones para satisfacer sus propias necesidades» (CMMAD, 1992). Esta definición de sustentabilidad incluye dos ideas clave: la de «necesidad» y la de «limitación», definida esta última como los límites impuestos — según el estado de la tecnología y la organización social en cada momento histórico- a la capacidad del medio ambiente para cumplir las necesidades de las generaciones presentes y futuras ${ }^{3}$. El citado Informe Brundtland dota definitivamente de un carácter político a la solución de los problemas ambientales al afirmar la necesidad de adoptar rápidas y decisivas acciones políticas con el fin de prevenir la destrucción ambiental (Brown, 1990; Lemons, 1995: 2-3). En realidad, el discurso sobre la sustentabilidad puede verse como una respuesta a la escuela de los límites del crecimiento, escuela que, desde los años setenta, venía postulando que la inexorable presión del crecimiento económico sobre el mundo natural daría como resultado una catástrofe ambiental. Frente a esta visión alarmista, el enfoque de la sustentabilidad es más flexible, al señalar que los daños ecológicos ocurren cotidianamente, de una manera gradual y sobre unas tasas o límites ambientales variables (Luke, 1995).

3 Los requerimientos que propone el Informe Brundtland para un desarrollo sustentable son los siguientes: un sistema político que asegure una efectiva participación en los procesos de decisión; un sistema económico que genere beneficios y conocimientos técnicos bajo unas bases autosostenidas; un sistema social que aporte soluciones a las tensiones que provoca un desarrollo no armónico; un sistema de producción que respete la obligación de preservar las bases ecológicas en el proceso de desarrollo; un sistema tecnológico que aporte continuamente nuevas soluciones; un sistema internacional de intercambios bajo condiciones de sustentabilidad; y un sistema administrativo que sea flexible y capaz de autocorregirse (O’Riordan, 1993: 53). 
El objetivo de lograr un desarrollo sostenible, tal como fue definido en el Informe Brundtland, ha sido ampliamente aceptado, habiendo sido, incluso, aprobado por los líderes del grupo de los siete países más industrializadas (G7) en la cumbre de Toronto de 1988. Asimismo, se considera como un principio igualmente válido en el que basar las políticas de desarrollo aplicables al Primer y Tercer Mundos, habiendo sido adoptado, además, por los distintos agentes económicos y sociales y por las organizaciones ecologistas, introduciéndose, así, no sólo en los medios de comunicación, sino también en los foros académicos y políticos.

En diciembre de 1989, la Asamblea General de Naciones Unidas, dando respuesta a los problemas identificados por el mencionado Informe Brundtland, convoca una reunión internacional, que finalmente tiene lugar en Río de Janeiro en 1992, con el nombre de Conferencia sobre Medio Ambiente y Desarrollo. En dicha Conferencia se aprueba la llamada Declaración de Río, en la que se alcanza un consenso político en cuanto a la finalidad y objetivos del desarrollo sostenible (Timoshenko, 1995). Resultado de esa Conferencia - también conocida como Cumbre de Río- ha sido la adopción de un programa de acción a largo plazo — denominado Agenda 21 - que pretende poner en práctica el concepto de desarrollo sostenible, dando un tratamiento integrado y equilibrado a las cuestiones ambientales y de desarrollo (Timoshenko, 1995) y respondiendo, así, a lo temas planteados en el Informe Brundtland. El propósito último de la Agenda 21 es la urgente modificación de las actividades humanas con el fin de que sean más respetuosas con el medio ambiente.

Después de más de veinte años de debates y acciones institucionales en relación al desarrollo sostenible, puede decirse que no existe una definición clara y universalmente admitida sobre el concepto de sustentabilidad (Hurrel y Kinsbury, 1992). En efecto, sustentabilidad y desarrollo sostenible son nociones que se interpretan de forma diferente según los puntos de partida y las metas que se pretendan conseguir. Sus significados suelen diferir entre ecologistas, economistas o planificadores ambientales, reflejando la variedad de sesgos disciplinarios, las diferencias entre paradigmas y las disputas ideológicas (Redclift, 1995).

Myers (1993) señala que la complejidad del concepto de sustentabilidad deriva de las distintas y simultáneas asociaciones que establece entre los problemas que trata de resolver. Según este autor, la sustentabilidad establece asociaciones del siguiente tenor: 1) entre diferentes problemas ambientales, es decir, entre un problema ambiental y otro; 2 ) entre diferentes esferas de la actividad humana, como la protección ambiental y el desarrollo; 3) entre el mundo desarrollado y el mundo en desarrollo; 4) entre la generación presente y la futura; 5) entre la protección de los recursos naturales y las necesidades humanas básicas; 6) entre la ecología y la economía; y 7) entre la eficiencia económica y la equidad social.

Además de la complejidad que encierra en sí mismo el propio concepto, su 
dificultad para hacerlo operativo radica en el hecho de que los elementos que integran la sustentabilidad no son invariables, sino que varían conforme cambian las demandas e interacciones entre los grupos que componen una determinada sociedad. Es, por tanto, un concepto histórica y socialmente determinado, es decir, un concepto abierto y adaptativo a las condiciones sociales en que se produce (Barbier, 1989). En cualquier caso, puede señalarse que la sustentabilidad es un concepto multidimensional, que contiene e integra variados elementos, tanto ecológicos como sociales (Pierce, 1992: 222). Su ambigüedad y escasa concreción es considerada en algunos círculos de opinión como la clave de su potencialidad (Redclift, 1992), mientras que en otros lo que se le critica es precisamente esa falta de claridad en su definición, señalándose que tal ambigüedad constituye uno de los principales obstáculos para abordar una estrategia viable y realmente operativa de desarrollo sostenible (Trigo, 1991).

Para algunos autores, el valor del concepto de sustentabilidad radica en su capacidad para superar las tradicionales divisiones entre, de un lado, políticas e intereses económicos y, de otro, políticas e intereses ambientales, dejando sin sentido el antagonismo entre progreso económico y protección del medio ambiente. Es decir, el concepto de desarrollo sostenible vendría a significar una especie de puente entre grupos sociales con posiciones y actitudes contrapuestas respecto a los problemas ambientales, o entre grupos que encontrarían en la propia ambigüedad del término de sustentabilidad un espacio común para cooperar entre ellos (Murdoch, 1993). No obstante, otros autores, como O'Riordan (1993), opinan que el concepto de sustentabilidad tiene «dos caras» y que, si bien puede constituir un puente entre grupos con distintos intereses, su ambigüedad también puede servir para favorecer la continuidad de prácticas tradicionales poco respetuosas con el medio ambiente.

Independientemente de las valoraciones que se hacen sobre la utilidad del concepto, lo cierto es que en la definición de la sustentabilidad se contemplan factores procedentes de distintas disciplinas y áreas de conocimiento. Autores como Daly y Gayo (1995) consideran tres aspectos combinados de la sustentabilidad, a saber: sustentabilidad ecológica, sustentabilidad económica y sustentabilidad social. La sustentabilidad ecológica se refiere al objetivo de mantener aquellas características de los ecosistemas que son esenciales para su supervivencia a largo plazo. La sustentabilidad económica implicaría una gestión adecuada de los recursos que justifique la continuidad del sistema económico vigente. Por último, la sustentabilidad social se alcanzaría cuando los costes y beneficios del desarrollo fuesen distribuidos de manera equitativa entre la población actual (equidad intrageneracional), pero teniendo en cuenta el bienestar de las generaciones futuras (equidad intergeneracional).

Centrándose también en estos tres aspectos, Atkinson (1995) señala que la sustentabilidad se alcanzaría cuando, combinándolos, pudiera transmitirse a las generaciones futuras un bienestar igual al menos al actual. Para hacer operativa esta idea y a efectos prácticos, este mismo autor utiliza el concepto de «capital» dividiéndolo en tres categorías, a saber: a) capital manufacturado (maquinaria, 
edificios, carreteras...); b) capital humano (conocimientos y habilidades), y c) capital natural (recursos naturales y medio ambiente). No obstante, según como se combinen estas tres categorías para alcanzar la sustentabilidad, se estaría ante dos escuelas del desarrollo sostenible: una "débil» y otra «fuerte» (Atkinson, 1995; De Andrés y Urzainqui, 1995).

La escuela de la sustentabilidad débil considera que es relativamente fácil y viable sustituir capital natural por capital manufacturado y/o capital humano, dado que estas tres categorías de capital contribuyen al bienestar general de un modo equivalente. De ello se deduce que lo que debe conservarse no es el valor de un bien determinado, sino un agregado de riqueza (Hartwick, 1978; Solow, 1986), al poderse sustituir completamente las distintas categorías de capital entre sí. Por su parte, la escuela de la sustentabilidad fuerte señala que algunos activos de capital natural son esenciales para el bienestar y la supervivencia de la especie humana a largo plazo (capital natural crítico), por lo que no son sustituibles por ninguna otra categoría de capital, sea éste manufacturado o humano.

Estas dos escuelas de la sustentabilidad pueden relacionarse con otros tantos enfoques a la hora de proponer soluciones para alcanzar un desarrollo sostenible, enfoques basados o bien en la tecnología o bien en la ecología. Así, existe, en primer lugar, un enfoque "tecnocéntrico", próximo al de la sustentabilidad "débil», que se caracterizaría por una fe ilimitada en la tecnología como medio para solucionar el problema de la escasez de recursos. Dentro de este enfoque se podría distinguir, a su vez, entre una posición que podríamos llamar "cornucopiana», que admite la posibilidad de una sustitución completa entre capital natural y capital humano o manufacturado, y una posición "acomodativa o de economía ambiental», según la cual la sustitución entre esas tres categorías de capital no puede ser nunca completa, planteándose entonces la necesidad de introducir en las estrategias de desarrollo los costes sociales de la degradación ambiental que puedan generar.

En segundo lugar, existe el enfoque "ecocéntrico», próximo a la escuela «fuerte» de la sustentabilidad, que enfatiza la necesidad de establecer límites a las actividades humanas, optando por un cambio de modelo de desarrollo económico para evitar problemas mayores. En el extremo más radical de este enfoque se sitúa la posición denominada "economía del estado estacionario", siendo más moderada la posición «comunalista», que propone introducir los principios de la termodinámica dentro de la ciencia económica.

La dialéctica entre "ecocentristas» y "tecnocentristas», como apunta O’Riordan (1993), se basa en tres dilemas que son inherentes a todas las sociedades: 1) El deseo de transformar la naturaleza con el fin de tener mayor certidumbre en el suministro de bienes naturales, frente a la necesidad de conservar el mundo natural a fin de asegurar la supervivencia. 2) Una preferencia por mejorar a corto plazo las condiciones de vida de la población promoviendo un incremento en el uso de los recursos naturales, frente a una preocupación de más largo plazo por los efectos desfavorables de dicho aumento en el consumo 
de recursos al poner en peligro la viabilidad futura del modelo económico. 3) La creencia en que la eficiencia, como forma de valorizar las inversiones, constituye la más apropiada directriz económica, frente al reconocimiento de que el principio de equidad debe contrarrestar tal objetivo de maximización (dilema eficiencia versus equidad).

Sobre estos tres dilemas se desarrolla una continua negociación entre «ecocentristas» y «tecnocentristas». Sin embargo, no parece que sean puntos antagónicos los que componen tales dilemas, sino más bien complementarios, lo que hace que todos ellos deban estar equilibrados en una sociedad sostenible ${ }^{4}$. Para O'Riordan (1995), cuando en una determinada sociedad los tres dilemas, o alguno de ellos, resultan irreconciliables es señal de que no se producen las condiciones necesarias para la sustentabilidad, si bien señala este autor que es posible la existencia de fases de estabilidad alternando con otras de tensión. En realidad, "ecocentristas" $y$ "tecnocentristas» se alinean con nociones de sustentabilidad diferentes, los primeros con la denominada sustentabilidad débil y los segundos con la sustentabilidad fuerte.

Algunos autores, como O'Riordan (1993), han analizado el tema de la sustentabilidad desde un punto de vista sociopolítico, proponiendo cinco condiciones para alcanzarla, a saber: a) una forma de democracia que trascienda el marco del Estado-nación; b) un sistema jurídico que garantice los derechos civiles y la justicia social, que sea capaz de promover un uso equilibrado de los recursos y que valore los derechos intrínsecos de la naturaleza; c) un procedimiento para sustituir los regímenes políticos cuyas acciones puedan desembocar en una mayor fragilidad del país en términos ambientales; d) la eliminación del principio de explotación; y e) el establecimiento de una variedad de mecanismos no gubernamentales para la distribución y gestión de recursos en áreas y comunidades con necesidad de ello. Se combinan así criterios éticos, morales, políticos y sociales, que también han sido admitidos desde otras disciplinas.

Desde una perspectiva económica, Jacobs (1991) indica que existen tres elementos clave para alcanzar la sustentabilidad: 1) la integración de consideraciones ambientales en la política económica; 2) el desarrollo del concepto de equidad (intra e intergeneracional), y 3 ) la redefinición del crecimiento económico de manera que incluya la calidad ambiental. Tales elementos derivan de una preocupación ética por el medio ambiente y las generaciones futuras, de modo que, para alcanzar la sustentabilidad, no sólo sería preciso realizar modificaciones en los sistemas físicos, sino también en los valores y actitudes sociales, tanto desde una perspectiva sociopolítica como económica (Atchia et al., 1995).

Lowe (1988) introduce otro aspecto más en el análisis de la sustentabilidad

${ }^{4}$ En opinión de Redclift (1991), las tres condiciones para el desarrollo sostenible (puntos de conflicto) serían el mantenimiento de las necesidades humanas o el mantenimiento del crecimiento económico o la conservación del capital natural, o las tres conjuntamente. 
al considerar que el desarrollo sostenible es un sistema de valores que opera al nivel del individuo y que, al situarse fuera de las prácticas institucionales, suele ser ineficaz. Por ello, la generalización de unos determinados valores sobre la sustentabilidad en el seno de la sociedad civil sería una condición necesaria, pero no suficiente, para introducir prácticas de desarrollo sostenible. De hecho, los poderes públicos, es decir, el Estado, ante la escasa vertebración de las demandas planteadas desde la sociedad civil, pueden retrasar o alterar aquellas propuestas de actuación que contengan prácticas sostenibles.

Otro aspecto que contribuye a la complejidad del desarrollo sostenible y a su dificultad para hacerlo operativo es el problema de la escala geográfica, problema éste que se plantea en dos niveles. En primer lugar, en el nivel de la interacción entre las soluciones planteadas a problemas ecológicos particulares (microecológicos) y las que se refieren a problemas ecológicos más generales (macroecológicos) (Lemons y Morgan, 1995: 99). En segundo lugar, en el nivel de la definición de los diferentes ámbitos sociales o territoriales de actuación, ámbitos en donde las metas de la sustentabilidad pueden ser incompatibles, de modo que lo que es sostenible en un ámbito puede no serlo en otro (Redclift, 1991: 37). Por ello, como se indicará en un próximo apartado, la escala de sustentabilidad es especialmente relevante a la hora de enjuiciar la sustentabilidad de un determinado sistema o subsistema, sobre todo en lo que se refiere a la agricultura.

Diversos autores han apuntado que el nivel de sustentabilidad más apropiado — tal vez el único posible- es el local (Martell, 1994; Agyeman y Evans, 1994; Rucht, 1993), ya que, argumentan, sólo sería a esta escala donde las propuestas de sustentabilidad se hacen suficientemente operativas para su puesta en práctica y donde pueden ser asumidas realmente por los habitantes de las comunidades implicadas. Desde esta perspectiva, el debate sobre la sustentabilidad ya no sería sobre la escala, al quedar claro que la idónea es la local, sino sobre la necesidad de establecer o no mecanismos de coordinación entre las distintas comunidades locales. Por ejemplo, en una sociedad descentraliza$\mathrm{da}$, donde diversas comunidades locales hayan escogido pautas de comportamiento sostenible, no sería preciso establecer mecanismos específicos de coordinación, dado que, de forma espontánea, se produciría la sustentabilidad entre aquellas unidades que, voluntariamente, siguieran pautas ambientales similares (Martell, 1994). No obstante, y desde una posición más realista, se asume que es necesario fijar algún tipo de mecanismo y pautas de coordinación - adaptados al origen, carácter y efectos de los problemas ambientales-, debiéndose, en consecuencia, iniciar un proceso de institucionalización en unidades de mayor dimensión. Sólo desde este punto de vista sería posible, en definitiva, abordar problemas de compensación entre comunidades por los recursos utilizados en el proceso de sustentabilidad.

Como conclusión a este primer apartado, puede decirse que la sustentabilidad se define de una forma bastante vaga e imprecisa tanto en sus aspectos sociales y políticos como en los geográficos o de escala. Ello hace que se plan- 
teen con poca precisión todavía las condiciones sociales que se requieren para alcanzar un desarrollo sostenible, y que no sea fácil medir sus posibles efectos. La inclusión, como hacen algunos especialistas, de elementos de orden ético (valores, justicia social y territorial...) entre las condiciones necesarias de la sustentabilidad hace aún más difícil, si cabe, la viabilidad de las soluciones propuestas, sobre todo a la hora de trasladarlas al terreno de las políticas públicas. A continuación analizaremos la introducción del concepto de sustentabilidad en un área concreta, cual es la de la agricultura, cuyas implicaciones de carácter medioambiental convierten a la actividad agraria en un interesante caso para ilustrar las dificultades de operativizar dicho concepto y para tratar el problema ya mencionado de la escala a la hora de aplicar acciones destinadas a lograr un desarrollo sostenible.

\section{LA SUSTENTABILIDAD EN LA AGRICULTURA}

Como es conocido, el modelo intensivo de agricultura ha alcanzado un nivel tal de especialización y generación de excedentes que cada vez son mayores las críticas por los efectos negativos que sus métodos y prácticas agrícolas y ganaderas ocasionan sobre el medio ambiente. En este contexto de excedentes agrícolas y de creciente deterioro ambiental hay que situar la aparición de los debates en torno al concepto de sustentabilidad en la agricultura, un concepto que, como en el debate sobre los modelos de desarrollo, viene a reconocer, por un lado, el grave deterioro producido en la calidad y cantidad de los recursos naturales y ambientales y, por otro, la necesidad de una actuación de carácter político para evitar, o al menos mitigar, tales efectos ${ }^{5}$.

En todo caso, al igual que señalábamos al revisar las diferentes acepciones del término sustentabilidad, el concepto de agricultura sostenible suele tener distintos significados, no existiendo un acuerdo generalizado sobre su definición (Horlings, 1994; Faeth, 1994). Las definiciones que se pueden encontrar en la literatura son muy variadas, dependiendo de cuál sea la característica que se quiera destacar, variedad que, además, se ve abonada por el hecho de que el concepto de agricultura sostenible es un concepto dinámico, es decir, que puede cambiar en el tiempo y en el espacio. Por tanto, a la hora de utilizarlo se plantean dos problemas: un problema de definición y otro de aplicación a los diferentes sistemas agrícolas. Esto complica la elaboración de normas y medidas que puedan ser aplicables con carácter universal a cualquier situación, ya que la diversidad de situaciones existentes en la agricultura hace difícil ponerlas en práctica. Por ejemplo, una determinada labor agrícola puede ser conside-

5 La reciente introducción de programas agroambientales específicos, ligados a la reforma de la PAC, intenta promover cambios en las prácticas de agricultura intensiva para introducir métodos más respetuosos con el medio ambiente. Ver Whitby (1996) y, para el caso español, el artículo de E. Moyano y F. Garrido incluido en ese libro. 
rada como perjudicial al medio ambiente o no, dependiendo de las circunstancias locales y del momento en que se realice. Por tanto, al situar el concepto de sustentabilidad agraria dentro del marco de la sustentabilidad general (Buttel, 1992; Horlings, 1994), los problemas de definición puestos antes de manifiesto se reproducen ahora e incluso aumentan, de modo que dicho concepto se convierte también en un concepto complejo y confuso. Como se refleja en la figura 1, de acuerdo con la línea argumental mantenida en la primera parte del texto, la sustentabilidad agraria, al igual que la sustentabilidad general, emanaría de las metas políticas y sociales de una determinada población, condicionadas por aspectos políticos o economicos e incluso éticos fruto de la interacción de distintos grupos sociales, y todo ello, a su vez, influye en los criterios ecológicos que serían los que definirían los contornos — débiles o fuertes- de la sustentabilidad agraria y general.

FIGURA 1

Sustentabilidad y sustentabilidad rural

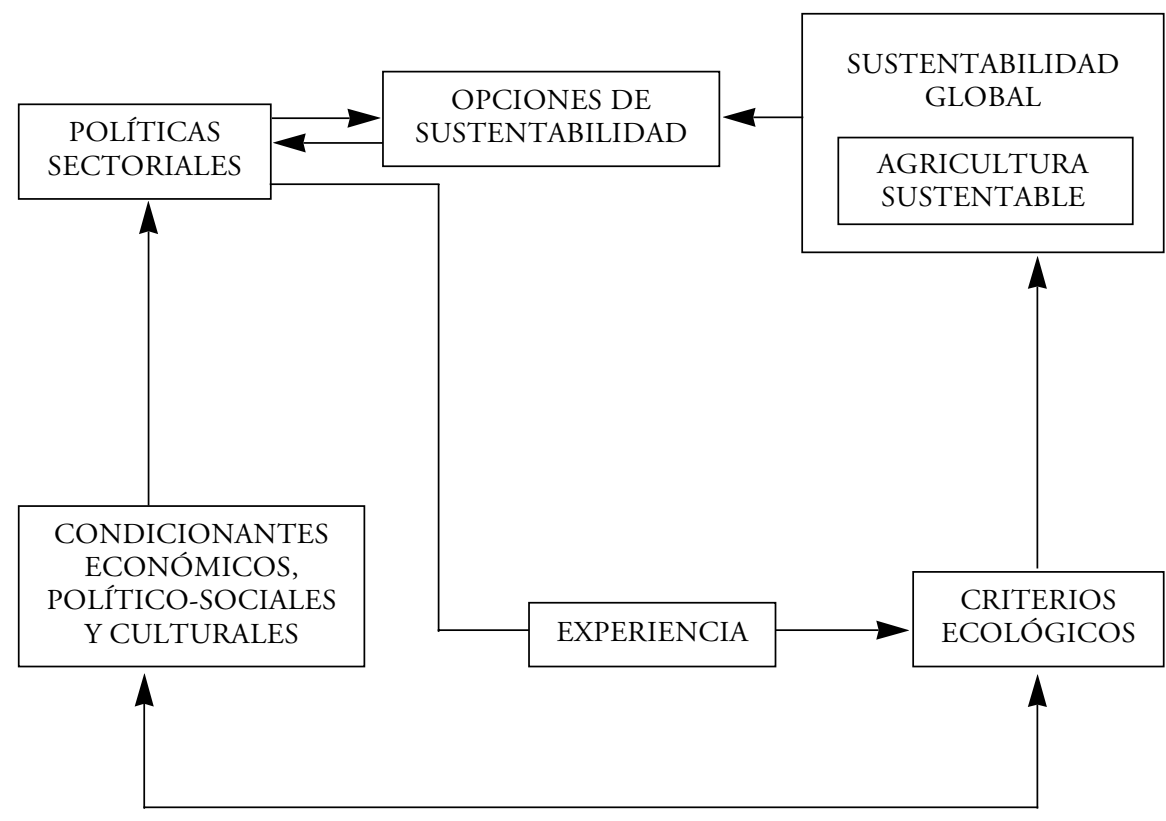

METAS POLÍTICAS O SOCIALES (grupos humanos)

FUENTE: Elaboración propia. 
Desde el mencionado Informe Brundtland (1987) se han construido al menos setenta definiciones del concepto de sustentabilidad, que enfatizan diferentes prioridades y metas: desde las definiciones que se centran en un enfoque exclusivamente económico o productivo a aquellas otras que incorporan los aspectos socioculturales y ecológicos. Por tanto, una definición universalmente admitida del concepto de agricultura sostenible no parece posible por ahora.

Las definiciones más elementales indican que con el concepto de agricultura sostenible se hace referencia a un tipo de agricultura que tenga menor dependencia respecto al uso y explotación de los recursos no renovables y que ocasione menor degradación ambiental en comparación con al actual sistema de agricultura convencional (Mannion, 1995: 331), refiriéndose también con dicho concepto al mantenimiento de la productividad agraria y de las rentas de los agricultores, y todo ello con el menor impacto ambiental posible (Faeth, 1994: 16). El defecto de estas definiciones tan genéricas reside en su carácter cualitativo, que las hace poco operativas, aspecto que ha sido utilizado por los economistas de los recursos naturales para establecer su crítica sobre ellas. Definiciones más elaboradas sugieren seis factores clave para sostener argumentalmente el concepto de producción alimentaria sostenible (Brklacich, Bryant y Smith, 1991) ${ }^{6}: 1$ ) contabilidad ambiental (identificación de los límites biofísicos); 2) productividad (producción por unidad de superficie); 3) capacidad de carga (máximo tamaño de la población que el medio ambiente puede soportar de una manera continuada); 4) viabilidad de la producción (viabilidad a largo plazo de las unidades de producción agrarias, bien explotaciones o grupos de explotaciones); 5) abastecimiento y seguridad en la producción (autosuficiencia en la producción alimentaria como clave de la estabilidad política y económica); 6) equidad (seguridad alimentaria intergeneracional, incluyendo una distribución equitativa de los alimentos).

Sin embargo, como apunta Mannion (1995), un análisis por separado de cada uno de estos factores ha puesto de manifiesto las dificultades de su medición y la diversidad de elementos de carácter físico, institucional, social e incluso psicológico que interviene en ellos. Esta diversidad pone de relieve las múltiples facetas de la relación hombre/medio ambiente en una de sus manifestaciones más características, como es el uso del suelo, sugiriendo, a su vez, el carácter holístico de este tipo de aproximaciones. Por otra parte, las diferentes argumentaciones de sustentabilidad pueden ser conflictivas entre ellas, debido tanto a las diferentes escalas que se utilicen como a criterios éticos (Reijntjes, Haverkort y Waters-Bayer, 1994: 3).

Una definición que recibió en su momento una importante adhesión por parte de la comunidad científica fue la propuesta por Gips (1984), que establece cuatro criterios o metas para la sustentabilidad en la agricultura: 1) ser ecológicamente sana, 2) económicamente viable, 3) socialmente justa y 4) tener

${ }^{6}$ Esta argumentación ha sido utilizada en España por Cadenas (1995). 
una escala humana. Estos criterios pueden ser aplicados, a corto o a largo plazo, a todos los aspectos de cualquier sistema agrícola ${ }^{7}$, desde la producción y la comercialización hasta la transformación y el consumo. Más que establecer qué métodos pueden ser empleados y cuáles no, esta definición ofrece cuatro criterios básicos sobre los cuales evaluar distintos modelos de agricultura y, si fuera necesario, modificarlos para crear sistemas agrarios sostenibles. En clara analogía con un modelo de programación lineal, se buscaría optimizar la sustentabilidad total del sistema agrario en cuestión, según el grado en que se pudieran satisfacer las cuatro metas establecidas, metas que estarían representadas por unas restricciones mínimas que el sistema debería satisfacer para ser sostenible. Este nivel de sustentabilidad no sería siempre el mismo, es decir, no quedaría fijado en el tiempo, sino que sería dinámico, de modo que la agricultura sostenible representaría una meta a alcanzar, pero nunca lograda.

Otros autores renuncian a definir de manera formal la noción de agricultura sostenible, limitándose a señalar que se refiere a un sistema agrario estructuralmente diferente del actual. Así, una agricultura sostenible implicaría un menor uso de inputs e introduciría nuevos métodos de gestión y nuevos sistemas de cultivo para posibilitar una mejor utilización de los recursos agrarios disponibles — sobre todo, a nivel local—. Los sistemas agrarios sostenibles deberían serlo a largo plazo en la productividad, ejerciendo, a corto plazo, una presión mínima sobre el medio ambiente y estando, además, caracterizados por una clara utilización de conocimientos y recursos locales (Doering, 1992: 22; Pretty, 1995: 8-9). En esta perspectiva, cualquier sistema de producción de alimentos debería cumplir unos objetivos de carácter ecológico y otros de carácter sociopolítico. Los primeros incluirían aspectos tales como una mayor incorporación de procesos naturales en los procesos agrícolas de producción, una menor utilización de inputs de carácter no renovable, un mayor aprovechamiento del potencial genético y biológico de las especies animales y vegetales, y el mantenimiento de un equilibrio entre modelos de producción, productividad potencial y límites de carácter ambiental, climático y paisajístico, a fin de asegurar a largo plazo unos determinados niveles de producción. Los objetivos de carácter sociopolítico abarcarían un acceso equitativo a los recursos productivos, el desarrollo de sistemas agrarios socialmente más justos, un incremento de la autodependencia entre agricultores y población rural y, por último, un desarrollo de sistemas de gestión integrados.

Combinando esos dos objetivos, algunos autores (Bowler, 1992) han sugerido la elaboración de una lista de prácticas compatibles con la agricultura sostenible, incluyendo aspectos como los siguientes: diversificación de usos del suelo; integración de agricultura y ganadería; rotación de cultivos; prácticas de agricultura orgánica; reciclado de nutrientes; escaso consumo de inputs de

Para un análisis comparado de la agricultura tradicional (con bajos inputs externos) y la agricultura convencional (con altos inputs externos o industrializada), sobre la base de los cuatro criterios dados por Gips, ver Gips (1984). 
energía y de productos agroquímicos... En relación con estas listas de prácticas agrícolas sostenibles, se han diferenciado diversos tipos de agriculturas que pudieran ser etiquetadas como "sostenibles»: agricultura de escasos inputs-outputs, agricultura ecológica, agricultura orgánica, agricultura alternativa, permacultura... (Pretty, 1995: 8). Algunos autores incluso han interpretado como sinónimos estas denominaciones, concediendo una dimensión únicamente semántica al problema. No obstante, la proliferación de estos vocablos para referirse todos ellos a prácticas y sistemas agrarios más respetuosos con el medio ambiente y la conservación de los recursos naturales ha servido también, en cierta medida, para aumentar la confusión en torno al tema de la sustentabilidad.

En todo caso, tal como apunta Neher (1992: 54), hay tres aspectos comunes a la definición de agricultura sostenible: productividad, calidad ambiental y viabilidad socioeconómica. Su conjugación y la importancia que se conceda a cada uno de ellos darían lugar a formas diferentes de expresar en la práctica la idea de agricultura sostenible. Por tanto, la agricultura sostenible podría establecerse como un concepto paraguas bajo el que se situaría toda una gama de sistemas y prácticas agrícolas que expresan esos criterios con distintos niveles o intensidades. Concebida en este sentido, la agricultura sostenible sería una meta a la que nos podríamos aproximar a través de numerosos caminos, llámense agricultura biológica, orgánica, ecológica, natural, regenerativa o de bajo input (Gips, 1984).

\section{Los niveles espaciales de la sustentabilidad agraria: el problema de la escala}

Anteriormente ya señalamos que algunos estudiosos de la sustentabilidad, como Redclift (1991), concedían especial relevancia al problema de la escala geográfica en el análisis del fenómeno. Referido al caso de la sustentabilidad agraria, la consideración de diversos niveles espaciales o escalas obedece, en primer lugar, a la importancia que algunos autores han concedido (Van Mansvelt, 1988) a los efectos a largo plazo de las interacciones e interdependencias que se producen dentro de cada nivel o (sub)sistema y entre los distintos niveles. Y, en segundo lugar, a la distinta potencialidad que, según sus características agroecológicas, cada área geográfica tiene para alcanzar las metas de la sustentabilidad.

Así, se plantean cinco niveles o escalas para analizar la sustentabilidad en la agricultura: 1) A nivel de la explotación agraria, que es donde, a escala microeconómica, se decide la rotación de cultivos, las inversiones, etc. $\left.{ }^{8} .2\right)$. A nivel de la comunidad local, constituida por agricultores relacionados entre sí por proximidad geográfica y vínculos culturales y sociales. 3) A nivel del paisaje, que es donde se manifiestan efectos tales como la degradación y deterioro pai-

${ }^{8}$ Ciertos autores (Van Mansvelt, 1988: 43) distinguen un nivel previo al de la explotación agraria, denominado de "campo cultivado" o "unidad de cultivo", que sería un sistema de carácter agronómico en donde los problemas están relacionados con el incremento de la productividad. 
sajísticos, la intensidad de los procesos erosivos, la pérdida de identidad de regiones naturales, etc. 4). A nivel nacional o regional, que es donde se manifiestan, a nivel macroeconómico, problemas tales como el aumento de costes debidos a la presión ambiental (contaminación de agua, suelo y aire) o el incremento de los costes sociales ocasionados por la producción agrícola y la generación de excedentes (impuestos, subvenciones). 5) A nivel internacional o global, que es donde se expresan los problemas Norte-Sur, el cambio climático, la deforestación y desertificación, el agotamiento de recursos, etc.

En esta secuencia de niveles, y conforme subimos en la escala geográfica, tienden a desaparecer los problemas de tipo técnico, siendo sustituidos por otros más bien de carácter sociopolítico y cultural (fig. 2). Asimismo, a nivel de la comunidad local o de la explotación es posible establecer acuerdos entre los diferentes agentes para fijar criterios de evaluación de las tendencias o "comportamientos» sostenibles. Pero en niveles superiores, como paisajes, regiones o Estados, se incrementan las dificultades para establecer cualquier medida de sustentabilidad, principalmente por los problemas para llegar a acuerdos entre los distintos agentes sociales, y se llegan a adoptar medidas de sustentabilidad débil. Como se pone de relieve en la figura 2, la sustentabilidad espacial vertical queda definida a través de diversos ejes cuyas caracteríticas están condicionadas por el nivel espacial en el que se introducen medidas o regulaciones que favorezcan la sustentabilidad. En todo caso, tales medidas adoptadas a distintos niveles no serían excluyentes, sino que podrían ser, o necesitarían ser, complementarias.

FIGURA 2

\section{Factores de sustentabilidad vertical}

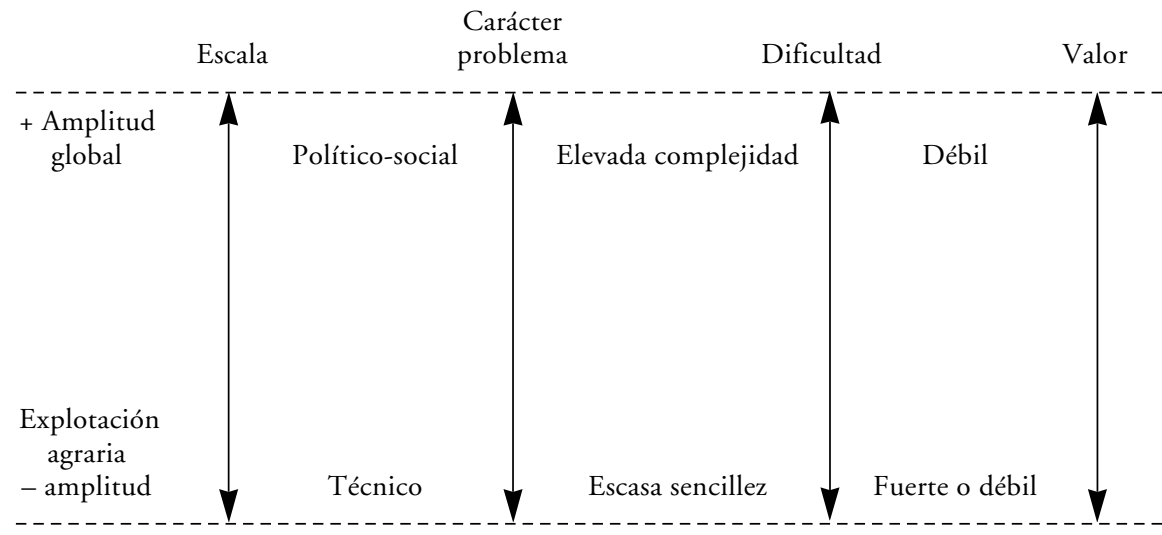

FUENTE: Elaboración propia. A.P.M. 
Cabe preguntarse, por tanto, ¿a qué nivel es más factible que se produzca la sustentabilidad? Es de reconocimiento casi unánime que la iniciativa debe producirse entre los agricultores y al nivel de la explotación agrícola, aunque las directrices puedan elaborarse a nivel regional o nacional (la UE entraría dentro de este nivel). En el propio Programa 21 de Naciones Unidas (Conferencia, 1993: 231) se indica que «... un planteamiento centrado en el agricultor es la clave para el logro de la agricultura sostenible tanto en los países desarrollados, como en los países en desarrollo...». Un eslabón intermedio lo constituiría la región o el paisaje («sistema» en términos económicos), donde «... los problemas ambientales se experimentan en un espacio dado, en relación con contextos físicos y económicos particulares, y afectan a poblaciones específicas...» (Bodiguel y Buller, 1991: 56). En este eslabón se engarzarían las directrices de niveles superiores con las iniciativas locales, aunque algunos autores afirman que la sustentabilidad agraria a nivel global es difícilmente alcanzable (Mannion, 1995: 330). Como se deduce de la figura 3, la sustentabilidad débil principalmente obedecería a la amplitud y heterogeneidad del marco geográfico y a la complejidad de la organización social. Mientras que a nivel de implantacion existiría un mayor número de actores individuales, que pueden acoptar medidas de sustentabilidad fuerte, pero que precisan la cohesión de alrededor de estructuras locales para que consigan sus efectos ecológicos y sociales.

\section{FIGURA 3}

\section{Componentes de las escalas de sustentabilidad en la agricultura}

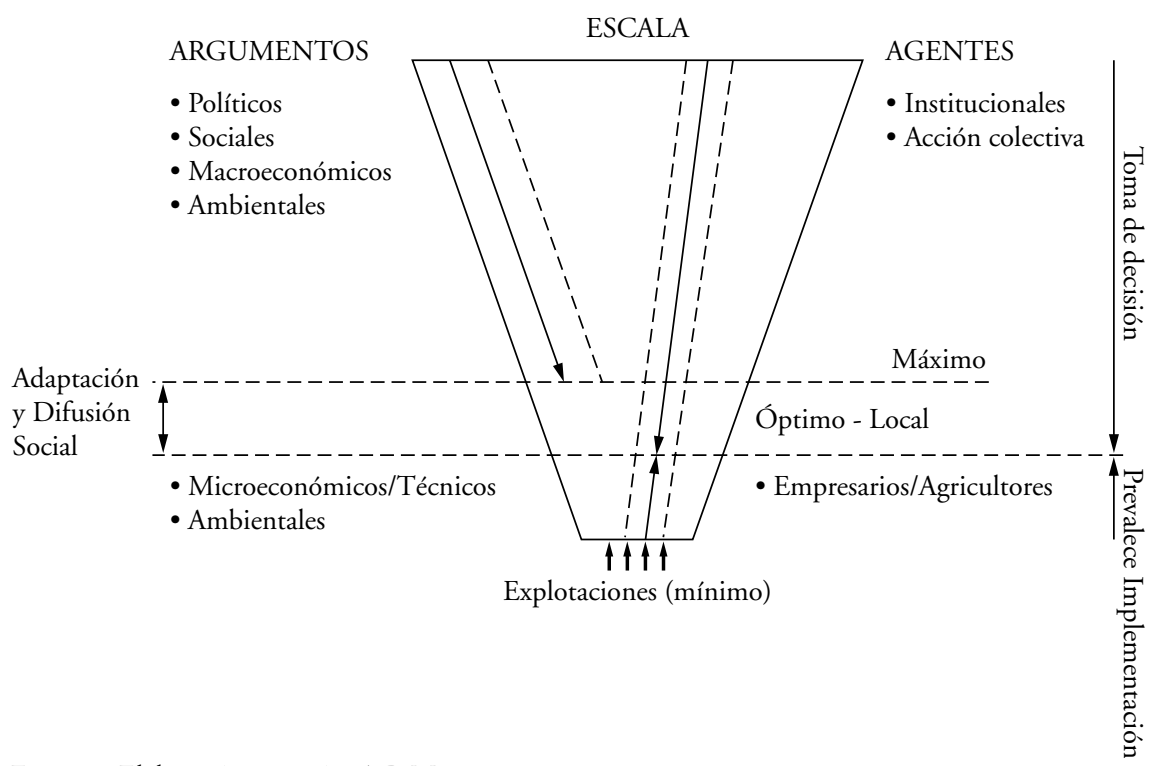

FUENTE: Elaboración propia. A.P.M. 
El desarrollo de prácticas sostenibles a nivel de explotación agraria requeriría, no obstante, dos condiciones. Por un lado, la participación de los agricultores en la puesta en marcha de tales prácticas y, por otro, la motivación del mayor número posible de ellos para coordinar la gestión de sus recursos. Ello significaría que el desarrollo de prácticas sostenibles no podría llevarse a cabo por agricultores aislados en su contexto local-paisajístico, debido a dos causas principales: en primer lugar, a que los costes externos de la degradación de recursos serían transferidos desde los agricultores que realizasen prácticas convencionales a aquellos otros agricultores que desarrollasen prácticas sostenibles; y, en segundo lugar, a que los beneficios ambientales producidos por un agricultor que de forma aislada realizase prácticas sostenibles en un área de métodos convencionales pueden ser muy reducidos o incluso insignificantes (Pretty, 1995: 22).

Por tanto, aunque es cierto que en la puesta en marcha de una agricultura sostenible tienen gran incidencia las motivaciones, conocimientos, cualificación y aspiraciones de los agricultores en el plano individual, su efectividad sólo se alcanza al nivel de comunidades o grupos de agricultores que actúan de forma coordinada. Así, en las estrategias contempladas en el ya citado Programa 21 se advierte que «... la descentralización de la adopción de decisiones hacia organizaciones locales y comunitarias es la clave para modificar el comportamiento de la población y aplicar las estrategias propias de una agricultura sostenible..." (Conferencia, 1993: 231). En consecuencia, se plantea en dicho programa la necesidad de promover la descentralización en el proceso de adopción de decisiones mediante la creación y fortalecimiento de las organizaciones locales. A este respecto, Pretty (1995) señala la importancia de la actuación al nivel de la comunidad local a través de instituciones y grupos de productores y usuarios, distinguiendo seis tipos (operativos a nivel local y regional) en función de las necesidades de la agricultura sostenible: 1) organizaciones locales, en general; 2) grupos para la gestión de los recursos naturales; 3) grupos de investigación agraria; 4) grupos de extensión rural; 5) grupos de gestión del crédito agrícola; y 6) grupos de consumidores.

Para este autor, la generalización de prácticas sostenibles exigiría no sólo que se conozcan entre la población los beneficios que tales prácticas pudieran comportar para la sociedad —es decir, su difusión como valor social—, sino también que existan grupos formalmente dentro de la comunidad local capaces de coordinar su gestión y aplicación. Esta segunda exigencia no es fácil de satisfacer, dado que las cuestiones relacionadas con el medio ambiente encuentran serias dificultades para incorporarse al discurso de los grupos organizados de agricultores, debido, en primer lugar, al lenguaje científico en que suele plantearse el tema de la sustentabilidad, circunscribiendo el debate a círculos restringidos, $y$, en segundo lugar, a la hegemonía del discurso productivista dentro de las organizaciones profesionales agrarias (Moyano, 1992).

En definitiva, el problema de la escala o ámbito territorial de la sustentabilidad agraria se resumiría en la necesidad de considerar tres niveles o escalas ordenadas verticalmente (fig. 3): 
1) Local-comarcal, como escala operativa donde efectivamente se produce la aplicación de las prácticas agrarias sostenibles mediante la coordinación de los comportamientos y actitudes de los agricultores. El marco de implantación presentaría un gap de mayor o menor amplitud en relación al carácter convergente o no de las estructuras político-administrativas regionales con las efectivas demandas e inquietudes locales (fig 3).

2) Regional-nacional, como escala decisional donde se plantean las directrices necesarias para el fomento de la sustentabilidad agraria y se adoptan las políticas públicas correspondientes.

3) Global-internacional, como escala de concertación entre grandes áreas geográficas, como países en desarrollo y países desarrollados, en donde se debaten los problemas macroecológicos del planeta y se intentan alcanzar acuerdos que guíen las distintas políticas nacionales.

También es posible utilizar en la definición de sustentabilidad agraria el potencial agroecológico de cada área (Redclift, 1990). De esta forma, se abordaría la sustentabilidad agraria desde una perspectiva horizontal en la que sería posible distinguir tres áreas: a) áreas de escasos recursos; $b$ ) áreas de agricultura intensiva; c) áreas forestales. En cada una de estas áreas existiría un potencial de sustentabilidad diferente y, por consiguiente, el desarrollo sostenible tendría distintos objetivos. En el primer caso, el desarrollo sostenible debería promover la conservación de recursos para mantener las condiciones básicas de vida. En el segundo caso, se debería mantener una elevada producción con mínimos costes ambientales. Y en el tercer caso, se debería evitar la eliminación de recursos genéticos y la erosión, a la vez que se mantendrían las oportunidades sociales y laborales dentro de una estrategia de gestión. Este análisis sugiere que el concepto de desarrollo sostenible tiene diferentes implicaciones para la utilización de recursos naturales en diferentes áreas, y que, por tanto, la sustentabilidad y el desarrollo agrario en cada área obedecen en buena parte a las características específicas - y a los problemas ambientales concretos- de las distintas áreas geográficas. Esto vuelve a indicarnos que la identificación de cuáles son las prácticas sostenibles es una labor difícil, porque el impacto de cualquier actividad agraria es singular en cada lugar.

\section{Enfoques en la sustentabilidad agraria}

Es posible diferenciar tres grandes enfoques que, aunque perviven de forma independiente, suponen, en realidad, enfoques con un claro carácter acumulativo:

1) Un enfoque basado en la agroecología, que parte principalmente del concepto de "agroecosistema» y que ha sido utilizado para sistemas agrarios de escasos inputs externos. Desde este punto de vista, en el diseño y gestión de los sistemas agrícolas y ganaderos sería necesario analizar y comprender previamente el funcio- 
namiento del ecosistema respecto de los flujos de energía y nutrientes, así como las bases de su estabilidad y los mecanismos que regulan de las poblaciones que allí habitan (Altieri, 1995). Este tipo de enfoque se caracteriza por proponer prácticas tales como el uso de rotaciones de cultivo, el reciclado de nutrientes, la gestión integrada de las plagas, el control interno de los ciclos biológicos, la diversificación de cultivos, la integración de agricultura y ganadería, la alta eficacia en el uso de energía y el desarrollo de estrategias óptimas que enfaticen un máximo beneficio sin aumento de la producción (Pierce, 1993). En definitiva, bajo este enfoque se pretende buscar la integración de conocimientos culturales y ecológicos que conduzcan a la sustentabilidad del sistema.

2) Un enfoque basado en la economía de los recursos, que arguye que la sustentabilidad tiene una base ecológica que no puede ser definida de manera independiente a otros valores asociados, como la cultura, la tecnología y la economía. De esta forma, es posible indicar que diferentes sociedades tienen diferentes valores colectivos y que, además, la disponibilidad de tecnología concede diferentes posibilidades y caminos de sustitución de capital natural por humano9. Según este enfoque, un sistema alimentario sostenible sería aquel que de forma indefinida pudiera atender las demandas de alimentos con unos costes ambientales y económicos socialmente aceptables. Este segundo enfoque tiene la virtud de haber definido la sustentabilidad en términos de costes (Pierce, 1992). No obstante, el problema que persiste es qué costes son socialmente aceptables y cuál es la capacidad del mercado para reflejar estos costes. En todo caso, el enfoque de la economía de los recursos ha sido criticado por su carácter unidimensional (Pierce, 1993), dado que la rentabilidad no debería ser el único factor a considerar en las prácticas agrarias y que las decisiones para adoptar métodos de agricultura sostenible también están influenciadas por el nivel educativo, la calidad de los servicios de extensión, las tradiciones locales, los modelos de acceso a los recursos y la accesibilidad a los resultados de la investigación (Faeth, 1994).

3) Un enfoque de carácter más amplio, que define un sistema alimentario de producción sostenible como aquel que sea capaz de asegurar a largo plazo y de forma simultánea el mantenimiento de la calidad ambiental y unos beneficios sociales y económicos para todos los individuos y empresas que participan en el sistema productivo, y que, a la vez, pueda producir una cantidad de alimentos suficiente y socialmente accesible. La agricultura sostenible sería una opción que surge en el contexto de crisis de la agricultura convencional y, más concretamente, de la reestructuración de la agricultura en la Unión Europea. En este sentido, la agricultura sostenible constituiría un camino, entre los distintos posibles, para la reorganización del sector agrario como respuesta a una crisis de dimensiones más amplias ${ }^{10}$.

9 Esta aproximación, por tanto, parte de un concepto de sustentabilidad débil.

${ }^{10}$ Crisis que tendría dos dimensiones estrechamente relacionadas: una productiva y otra ambiental. 
Como anteriormente se ha indicado, no existe un único criterio para definir la agricultura sostenible. Se han citado tres (Neher, 1992: 54): ecológicoambiental, socioeconómico y productivo, a los que Bowler (1992) añade otros dos: presupuestario y político.

El criterio ecológico-ambiental sería preponderante sobre los demás y quedaría caracterizado por los siguientes aspectos: acercamiento holístico al sistema agrario; mantenimiento de la estabilidad del ecosistema; vigilancia de los recursos básicos; uso eficiente de la energía; conservación de la fertilidad del suelo mediante materia orgánica; ausencia de fertilizantes inorgánicos y productos agroquímicos... El criterio socioeconómico quedaría resumido en la capacidad de generar nuevos empleos en la agricultura después de décadas de pérdida de población activa agraria, y en la obtención de mejores precios para los productos de la agricultura sostenible, frente a los productos convencionales. El criterio productivo se cumpliría si la agricultura sostenible fuese capaz de alimentar a la población no agraria. En cuanto al criterio presupuestario, se querría indicar con él la necesidad de reducir la dotación de fondos públicos a la agricultura mostrando cómo el paso de una agricultura convencional a una sostenible no requeriría un aumento de las subvenciones al transferirse los costes de la conversión a los consumidores mediante un incremento de precios (el «precio-premio" por alimentos de mayor calidad). Finalmente, el criterio político se fundamentaría en la descentralización de los procesos de toma de decisiones para que puedan intervenir más directamente los productores y los consumidores. La combinación de estos cinco criterios sería requisito fundamental para catalogar un sistema agrario como sostenible, de modo que la ausencia de alguno de ellos significaría un menor grado de sustentabilidad.

\section{CONCLUSIONES}

La revisión conceptual del concepto de sustentabilidad nos lleva a concluir que términos como desarrollo sostenible, desarrollo agrario sostenible o agricultura sostenible no son conceptos unánimemente aceptados ni tienen el mismo significado para los distintos autores y actores sociales (más allá de un núcleo débil por todos compartido). Por el contrario, las diversas concepciones reflejan posiciones diferentes respecto a la cuestión ambiental en general y al desarrollo sostenible en particular, que a su vez son reflejo de diferencias ideológicas y de intereses.

En consecuencia, las respuestas de los distintos actores sociales implicados respecto a estos temas reflejan no sólo sus percepciones, posiciones e intereses, sino también la diversidad de contenidos en los discursos y propuestas que sobre estos conceptos (desarrollo sostenible, desarrollo rural sostenible y agricultura sostenible) emanan desde las distintas instancias. Así, las percepciones y actitudes de los agricultores y las organizaciones agrarias hacia la regulación ambiental de la agricultura (consecuencia práctica de la concepción de desarro- 
llo sostenible) dependerán de los contenidos o significados que se den a estos conceptos.

Por otra parte, se puede partir de la idea de que las demandas en favor de un desarrollo sostenible (y sus aplicaciones al medio rural y al sector agrario) emanadas de las distintas instancias oficiales y de agentes sociales externos al sector contienen, a veces, posiciones ambiguas o incompatibles entre sí, lo que puede contribuir aún más a la confusión en torno a la sustentabilidad. Las diversas concepciones muestran diferencias en cuanto a las diferentes dimensiones de los conceptos y las escalas analíticas y operativas del mismo. El análisis de las respuestas, actitudes y opiniones de los agricultores a la regulación ambiental en la agricultura o a la ambientalización de la misma dependerá, pues, de los contenidos de las diferentes acepciones del desarrollo sostenible y su dimensión agraria.

\section{ALGUNAS DEFINICIONES DE DESARROLLO SOSTENIBLE}

BARBIER: Nivel óptimo de interacción entre tres sistemas —el biológico, el económico y el social—, que se alcanzaría a través de un proceso dinámico y adaptativo de intercambios.

Punto de destino de todas las aspiraciones en materia de desarrollo deseable.

BARTELMUS: Es el desarrollo que mantiene un nivel de rentas determinado a base de conservar las fuentes de dichas rentas: el stock de capital natural y producido.

CIAR: Debería combinar el uso planificado de los recursos empleados en la producción agraria con los esfuerzos para mantener o fortalecer la base existente de recursos que prevenga la degradación medioambiental, para satisfacer las cambiantes necesidades de la sociedad.

$F A O$ : Es la gestión y conservación de los recursos naturales y la orientación del cambio técnico e institucional de forma que se asegure la continua satisfacción de las necesidades de las generaciones presentes y futuras.

HART: Se refiere al uso de los recursos biofísicos y económicos para obtener productos cuyo valor socioeconómico y medioambiental actual representa más que el valor de sus inputs, mientras que al mismo tiempo se protege la productividad futura del medio ambiente.

PEARCE: Cambio económico sujeto a la constancia del stock natural.

PEARCE et al.: El desarrollo económico sostenible implica maximizar los beneficios netos del desarrollo económico, que queda supeditado al mantenimiento a lo largo del tiempo de los servicios y la calidad de los recursos naturales.

ROBINSON et al.: Es la persistencia, sobre un futuro aparentemente indefinido, de ciertas características deseadas y necesarias del sistema sociopolítico y su medio ambiente natural. 


\section{DEFINICIONES DE AGRICULTURA SOSTENIBLE}

AGENCIA ALEMANA DE COOPERACIÓN TÉCNICA (GTZ): Es aquella que tenga el propósito de «establecer una productividad del suelo elevada y duradera y, por tanto, conservar o restablecer un medio ambiente bien equilibrado".

ALLEN et al.: Es aquella que de forma equitativa equilibra la preocupación por el medio ambiente, la viabilidad económica y la justicia social entre todos los sectores de la sociedad.

DOUGLASS: Los tres componentes necesarios de la sustentabilidad agraria son: la economía, la ecología y la comunidad (o sociedad).

FISHER: Componentes básicos para la sustentabilidad en la agricultura: dinamismo sistémico, armonía con la naturaleza, diversidad, recursos renovables, participación social, nutrición, comunidad y estética.

FRANCIS: Estrategia de gestión cuya meta es reducir los costes ocasionados por los inputs, minimizando el daño medioambiental, y suministrar producción y beneficio a lo largo del tiempo.

GIPS: Una agricultura sostenible es ecológicamente sana, económicamente viable, socialmente justa y humana.

KEENEY: Sistemas agrarios que son respetuosos con el medio ambiente, rentables, productivos y que mantienen el tejido social de la comunidad rural.

NATIONAL RESEARCH COUNCIL: Producción de alimentos y fibras que emplea estrategias ecológicas de producción para reducir inputs y daños ambientales, a la vez que se promueve una producción rentable, eficiente y a largo plazo.

PIERCE y BLKLACICH: En la agricultura sostenible deben cubrirse al menos tres criterios: 1) medioambiental (mantener o intensificar la base de recursos agrícolas); 2) socioeconómico (proporcionar recompensas económicas equitativas a los agricultores y a las comunidades rurales en el sector de producción); y 3) produccionista (producir una cantidad suficiente de alimentos).

SCHMIDT y GASTÓ: Hay que considerar los diferentes elementos que componen el ecosistema y buscar el equilibrio entre ellos: crecimiento económico, sustentabilidad y equidad.

WENDELL BERRY: La agricultura sostenible es aquella que no reduce ni el suelo ni la población

WILKEN: Consiste en obtener una producción continuada de los agroecosistemas dependiente del mantenimiento de los recursos en que se basa dicha producción. 


\section{BIBLIOGRAFÍA}

Agyeman, J., y Evans, B. (1994): "The new environmental agenda», en J. Agyeman y B. Evans, Local environmental policies and strategies, Londres, Longman, pp. 1-22.

ATCHIA et al. (1995): "National environmental management plans and programmes for sustainable development", en M. Atchia y S. Tropp (eds.), Environmental management. Issues and solutions, Chichester, Wiley, pp. 34-41.

Altieri, M. A. (1995): «El estado del arte de la agroecología, y su contribución al desarrollo rural en América Latina», en A. Cadenas (ed.), Agricultura y desarrollo sostenible, Madrid, MAPA, pp. 151-203.

AtKInSON, G. (1995): "La sostenibilidad como resiliencia en sistemas agroecológicos», en A. Cadenas (ed.), Agricultura y desarrollo sostenible, Madrid, MAPA, pp. 281-300.

BARbIER, E. (1989): Economics, Natural Resource Scarcity and Development, Londres, Earthscan Publications.

Bodiguel, M., y Buller, H. (1991): «L'agriculture et l'environnement en Europe», Ecodecision, 1, pp. 55-59.

BOWLER, I. R. (1992): «Sustainable agriculture as a path of farm business development», en I. R. Bowler, C. R. Bryant y M. D. Nellis, Contemporary rural systems in transition. Volume 1. Agriculture and environment, Wallingford, CAB Int., pp. 237-253.

Brklacich, M.; Bryant, Ch. R., y SMith, B. (1991): «Review and appraisal of concept of sustainable food production systems», Environmental Management, vol. 15, n. ${ }^{\circ}$ 1, pp. 1-14.

BROWN, S. (1990): «Humans and their environment: changing attitudes», en J. Silvertown y P. H. Sarre (eds.), Environment and society, Londres, The Open University, pp. 238-269.

ButTel, F. H. (1992): «Environmentalization: origins, processes and implications for rural social change», Rural Sociology, 57 (1), pp. 1-27.

CADENAS, A. (1995): «Conceptos y criterios operativos de sustentabilidad de sistemas de producción agraria, forestal y alimentaria», en A. Cadenas (ed.), Agricultura y desarrollo sostenible, Madrid, MAPA, pp. 71-90.

Comisión Mundial del Medio Ambiente y del Desarrollo (1992): Nuestro futuro común, Madrid, Alianza Editorial.

Commins, P. (1990): «Restructuring agriculture in advanced societies: Transformation, crisis and responses", en T. Marsden et al. (eds.), Rural restructuring: Global processes and their responses, London, David Fulton Publishers, 197 pp.

Conferencia de las Naciones Unidas sobre el Medio Ambiente y Desarrollo (1993): Río 92. Programa 21, Madrid, MOPT, $312 \mathrm{pp}$.

DalY, H. E., y GAYO, D. (1995): «Significado, conceptualización y procedimientos operativos del desarrollo sostenible: posibilidades de aplicación a la agricultura», en A. Cadenas, (ed.), Agricultura y desarrollo sostenible, Madrid, MAPA, pp. 19-38.

DE ANDRÉs, R., y URZAINQUI, E. (1995): "Comercio internacional agrario, GATT y desarrollo sostenible», en A. Cadenas, (ed.), Agricultura y desarrollo sostenible, Madrid, MAPA, pp. 361-384.

DoERING, O. (1992): «Federal policies as incentives or disincentives to ecologically sustainable agricultural systems», en R. K. Olson (ed.), Integrating sustainable agriculture, ecology, and environmental policy, Nueva York, The Haworth Press, pp. 21-36.

FAeth, P. (1994): «Building the case for sustainable agriculture. Policy lessons from India, Chile and the Philippines", Environment, vol. 36, n. ${ }^{\circ}$ 1, pp. 16-34.

GIPS, T. (1984): «What is sustainable agriculture», Manna, julio-agosto.

HARTwICK, J. M. (1978): «Investing returns from depleting renewable resource stocks and intergenerational equity», Economic Letters, 1, pp. 85-88.

HorLings, I. (1994): «Policy conditions for sustainable agriculture in The Netherlands», The Environmentalist, 14, 3, pp. 193-199.

Hurrel, A., y Kingsbury, B. (1992): "The international politics of the environment: an introduction", en A. Hurrel y B. KignsBury, The interntional politics of the environment. Actors, interests, and institutions, Oxford, Clarendon Press, pp. 1-50. 
JaCOBS, M. (1991): The green economy. Environment, sustainable development and the politics of the future, Londres, Pluto Press, $312 \mathrm{pp}$.

LEMONS, J., y MORGAN, P. (1995): "Conservation of biodiversity and sustainable development", en J. Lemons y D. A. Brown (eds.), Sustainable development: science, ethics, and public policy, Dordrecht, Kluwer ac. Pub., pp. 77-109.

LOWE, P. (1988): "Environmental concern and rural conservation politics», en M. Whitby y J. Ollerenshaw, Land-use and the european environment, Londres, Belhaven Press, pp. 6877.

- (ed.) (1992): «Industrial agriculture and environmental regulation», Sociologia Ruralis, 32 (1), pp. 1-118.

LOWE, P., y WARD, N. (1994): «Agricultura y medio ambiente: temario sociológico», Agricultura y Sociedad, n. ${ }^{\circ}$ 71, MAPA, pp. 257-270.

LUKE, T. W. (1995): "Sustainable development as a power/knowledge system: the problem of "governmentality"”, en F. Fischen y M. Black (eds.), Greening environmental policy. The politics of a sustainable future, Londres, Paul Chapman Pub. Ltd., pp. 21-32.

Mannion, A. M. (1995): Agriculture and environmental change: temporal and spatial dimensions, Chichester, John Wiley and Sons, 405 pp.

Martell, L. (1994): Ecology and society. An introduction, Oxford, Polity Press, 232 pp.

Mormont, M. (1994): "La agricultura en el espacio rural europeo», Agricultura y Sociedad, n. ${ }^{\circ}$ 71, MAPA, pp. 17-49.

MOYANO, E. (coord.) (1992): Las organizaciones profesionales agrarias en la Comunidad Europea, Madrid, Serie Estudios del MAPA.

Murdoch, J. (1993): «Sustainable rural development: towards a research agenda», Geoforum, 24, 3, pp. 225-241.

MYERS, M. (1993): «The question of linkages in environment and development», Bioscience, 43, pp. 302-310.

NeHER, D. (1992): «Ecological sustainability in agricultural sustems: definition and measurement", en R. K. Olson (ed.), Integrating sustainable agriculture, ecology, and environmental policy, Nueva York, The Haworth Press, pp. 51-61.

OCDE (1989): Agriculture and environmental policies. Opportunities for integration, París.

O'RIORDAN, T. (1993): "The politics of sustainability», en R. K. Turner (ed.): Sustainable environmental economics and management. Principles and practice, Londres, Belhaven Press, pp. 37-69.

- (1995): "Linking the environmental and social agendas», The Environmentalist, 15, pp. 233239.

PIERCE, J. T. (1992): "The policy agenda for sustainable agriculture», en I. R. BOWLER, C. R. Bryant y M. D. Nellis, Contemporary rural systems in transition. Volume 1. Agriculture and environment, Wallingford, CAB Int., pp. 221-236.

- (1993): "Agriculture, sustainability and the imperatives of Policy Reform», Geoforum, vol. 24, n. ${ }^{4}$, pp. 381-396.

Pretty, J. M. (1995): Regenerating agriculture. Policies and practice for sustainability and selfreliance, Londres, Earthscan, $320 \mathrm{pp}$.

RedCLIFT, M. (1990): «Developing sustainably. Designating agroecological zones», Land Use Policy, vol. 7, n. ${ }^{\circ}$ 3, pp. 202-216.

- (1991): "The multiple dimensions of sustainable development», Geography, 76, 330, pp. 3642.

- (1992): «La función de la tecnología agraria en el desarrollo sostenible», en P. LowE, T. Marsden y S. Whatmore, Cambio tecnológico y medio ambiente rural, Madrid, Serie Estudios MAPA, pp. 143-178.

- (1995a): "Values and global environmental change», en Y. Guerrier et al. (eds.), Values and the environment. A social science perspective, Londres, Wiley, pp. 7-18.

- (1995b): "Desarrollo sostenible: ampliación del alcance del debate», en A. Cadenas (ed.), Agricultura y desarrollo sostenible, Madrid, MAPA, pp. 39-70. 
Reijntjes, C.; Haverkort, B., y Waters-Bayer, A. (1994): Farming for the future. An introduction ot low-external-input and sustainable agriculture, Londres, MacMillan Press, $250 \mathrm{pp}$.

Rucht, D. (1993): "Think globally, act locally? Needs, forms and problems of cross-national cooperation among environmental groups», en J. D. Lieferink, P. D. Lowe y P. J. Mol (eds.), European integration and environmental policy, Londres, Belhaven Press, pp. 75-96.

Solow, R. M. (1986): "On the intergenerational allocation of natural resources», Scandinavian Journal of Economics, 88, pp. 141-149.

Timoshenko, A. S. (1995): "From Stockholm to Rio: the institucionalization of sustainable development», en W. LANG, Sustainable development and international law, Londres, Graham and Trotman, pp. 143-160.

TRIGO, E. J. (1991): Toward a strategy for sustainable agricultural development, Costa Rica, IICA.

VAN MANSVELT, J. (1988): "The role of lower-input technologies in the future», en M. WHITBY y J. Ollerenshaw, Land-use and the european environment, Londres, Belhaven Press, pp. 4253.

Whitby, M. (ed.) (1996): The European Environment and CAP reform. Policies and prospects for conservation, Wallingford/Oxon, CAB International.

\begin{abstract}
This paper have two differents parts. In the first study the complexity and the multiple dimensions of sustainability and sustanable development concepts. The perspective with referring to sustentability and sustainable development is that its very appeal is its vagueness. In the second part study the options of sustanaible rural development in a global perspective and propouse the different levels at which rural sustainability its important.
\end{abstract}


NOTAS DE INVESTIGACIÓN 